\title{
The design of new Intelligent Protector of Feeder Switch Based on Cortex-M3
}

\author{
Yaohui WU ${ }^{1, a}$, Tianbo $\mathrm{WU}^{1, \mathrm{~b}}$, Zheng Zheng ${ }^{1, \mathrm{c}}$ and Hua Wei ${ }^{2, \mathrm{~d}}$ \\ ${ }^{1}$ School of Electrical Engineering and Automation, Henan Polytechnic University, \\ Jiaozuo, 454000, Chain; \\ ${ }^{2}$ Jiaozuo water co., LTD, Jiaozuo, 454002, Chain

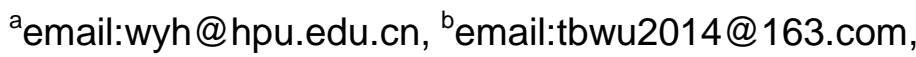 \\ cemail:zhengzh@hpu.edu.cn, ${ }^{\mathrm{e}}$ email:qf88866@163.com
}

Keywords: Feeder Switch; Integrated Protector; Cortex-M3; UC/OS-III; Graphical Interaction

\begin{abstract}
According to the continuous increasing intelligent demands of power supply equipments in digital mine and the current upgrading needs of the feeder switch integrated protector, this paper describes a new type of intelligent mineral low voltage comprehensive protector with graphical interaction. The protector takes the Cortex-M3 kernel chip as control core and adopts the embedded real-time operating system, UC/OS-III ,to switch and schedule the tasks. On the basis protective function of short-circuit, overload, phase failure, three-phase imbalance, leakage, over-voltage and under-voltage fault etc, the protector reaches the graphical display by UCGUI interface technology and realizes the touched intelligent human-computer interaction by the increased function as the touched digital keyboard.
\end{abstract}

\section{Introduction}

The intelligent technology of mining equipment plays an important role in the construction of digital mine, and the intelligent comprehensive protector of motor and feeder switch are the important guarantee for power supply security [1].This paper describes a new type of intelligent mineral low voltage comprehensive protector, which takes the Cortex-M3 kernel chip-STM32F103ZE- as control core. According to the characteristics and actual needs of power supply of coal mine, the protector can reach the protection for power supply system within the voltage grade at $380 \mathrm{~V}$ or $660 \mathrm{~V}$ or $1140 \mathrm{~V}$ and the current scope from 20A to 2000A.

\section{Hardware structure and Principle}

The peripheral circuit of protector including data acquisition, communication interface, power supply, switch input and human-computer interaction etc. The whole structure is shown in Fig.1.

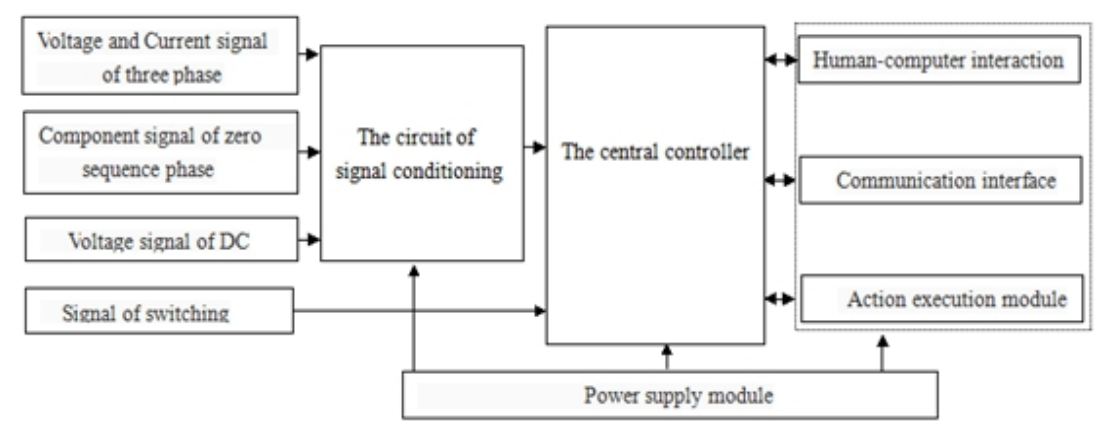

Fig. 1 The hardware structure diagram of protector

Before the system switch is closed, protector will call atresia protect determine whether the system can start. If the switch closed security, the signal conditioning circuit will synchronously turn the AC voltage and current of system into voltage signal. And the circuit adjusts the additional DC signal for DC voltage signal according to certain proportion. The protector use ADC to collect and process the signals, it compares the results with the set value of protection to judge running situation of system. 
The protector communicates with PC by RS485 and removes fault through the module for action execution and stores the fault information into external memory. While the staff can query fault and set parameter through the interface of human-computer interaction with touch functions.

\section{Design of the Software System}

The protector adopts the real-time operating system UC/OS-III to realize the reasonable management of system software [2].It can not only improve the protection of software architecture, but also can ensure real-time protection [3].The architecture of software is shown in Fig.2.

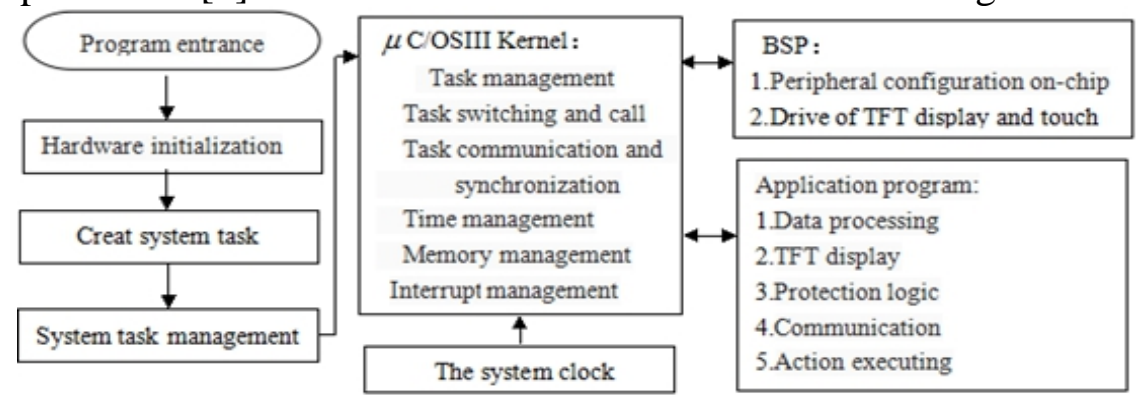

Fig.2 The software structure based on UC/OS-III

The main program calls BSP initialization function to provide a hardware foundation for system software before the system task operation. In order to realize the function, the protector needs to deal with at least five tasks. The tasks include signal process, TFT display, communication, protection logic and action executing etc.

The Task of Signal Process and Adopt. The comprehensive protector needs to collect a total of nine electric parameters. That include three-phase voltage, three-phase current, zero sequence voltage, zero sequence current and additional DC current for leakage protection. According to the types and characteristics of signals, the operation mode of ADC is configured to continuous scanning mode. The sampling time is set to 239.5 sampling period and the sampling number in a cycle of each signal is set to 95.The data processed by the designed program based on FFT algorithm [4].

The Protect Tasks of Protector. According to the requirements of "Safety Regulations in Coal Mine”, the switch should be ensuring active reliable while the faults occur. The faults include phase failure, short-circuit, overload leakage, over-voltage, three-phase imbalance and under-voltage fault etc. According to the type of protection, the protection program is divided into four types such as voltage protection, current protection, leakage protection and blocking protection.

The Protection of Voltage.This protection includes imbalance protection of voltage, over voltage and low voltage, the flow chart of task is shown in Fig.3.

While the differences value of any two phase reach to the setting value, protector display the state of voltage unbalance. The fault handling function will be called to deal with the problem, no matter whether the result is greater than the set value or less than the set value. The switch of system droved by the protector and the display of protector will be over-voltage or lower-voltage corresponding to the fault.

The Protection of Current.The protection includes imbalance protection of load, short circuit protection and overload protection, the flow chart of task is shown in Fig.4.

At first, while the valid value of any phase is greater than the set value for the quick break and the duration exceeded the set time, the program will step into the fault handling function to drive the switch and the display of protector is short circuit. The second, while the value of any phase is greater than rated current, micro controller will also drive the switch and display overload fault. The overload protection follows the principle of inverse time or definite time. In addition, when detect system current is zero, the fault handing function will be called to drive the switch and display phase failure. 
The Task of Leakage Protect. According to the principle of protection, the existing method is implemented by the detection of insulation impedance of transmission line. The protection program of leakage in this device includes the protection of zero sequence voltage and current, the protection of power direction and additional DC protection. The flow chart of task is shown in Fig.5.

The signals like zero sequence voltage, zero sequence current and additional DC is filtered by protector. It chooses the corresponding protection way according to the set working station like main switch or separate. When the system value reaches or exceeds the set value, it will drive the switch and display the fault of leakage.

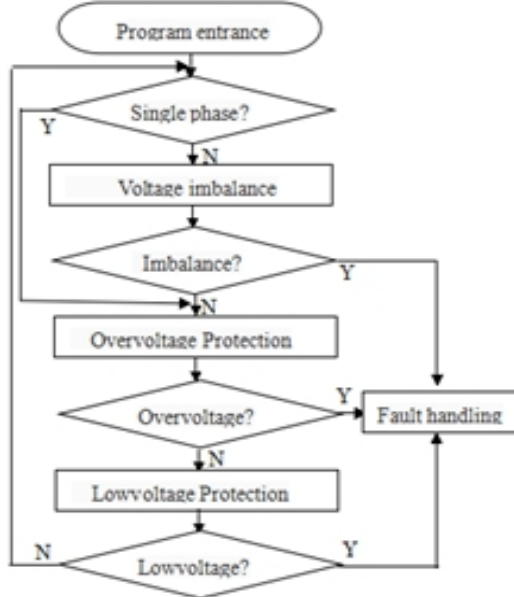

Fig.3 The protection chart of voltage

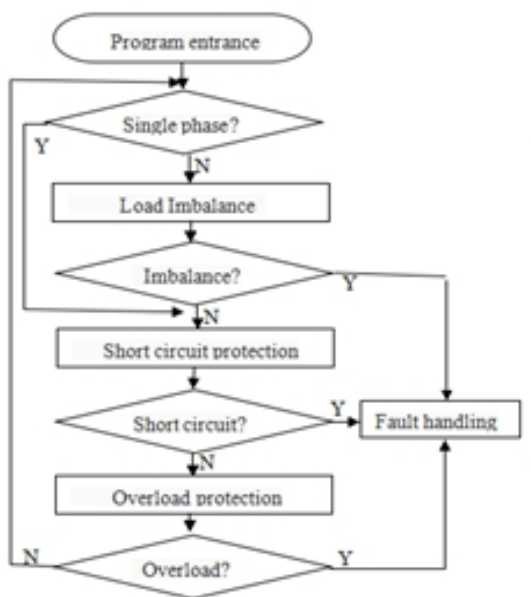

Fig.4 The protection chart of current

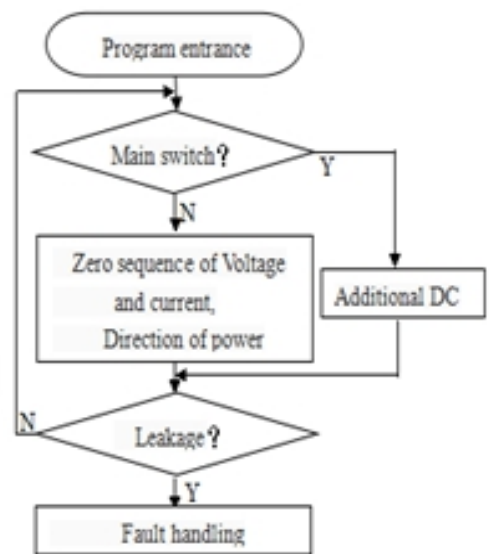

Fig.5 The protection chart of current

The Protection of Atresia .This protection includes mechanical atresia, leakage atresia and wind-power atresia. No matter any kind of problem appeared, the system will not be charged with electricity by protector. The problem include the mechanical door of switch is no closed or the hidden trouble of leakage exists in line or the fan did not open.

The Task of Human-computer Interaction .In order to improve the intelligent level of equipment, the driver of TFT display and touch transplanted to the UCGUI. The GB2312 character driven needed to add into the UCGUI architecture, because it does not support Chinese characters display [5].The program of task is shown in Fig.6.

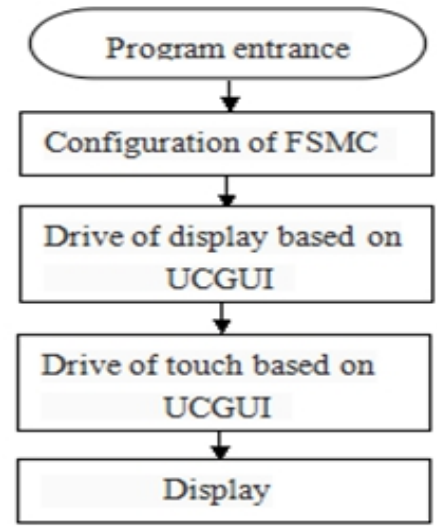

Fig.6 The interaction chart of Human-computer

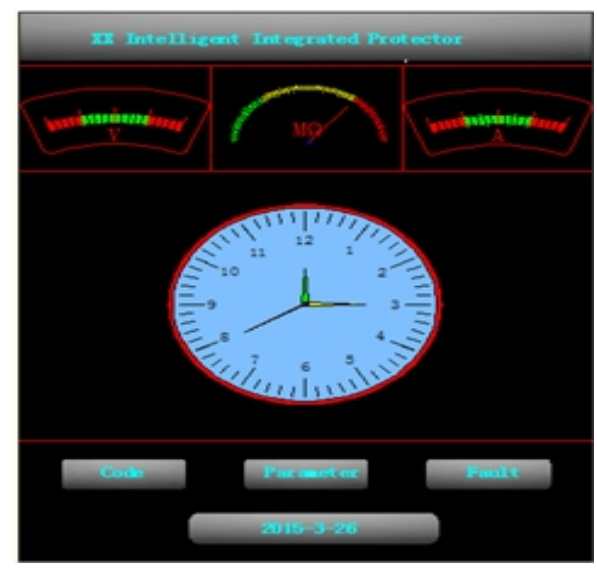

Fig.7 The chart of display

The screenshots is shown in Fig.7 display the system operation when the system voltage is $380 \mathrm{~V}$, current is 200 A. As shown in Fig.7, the voltage and current of system and insulation status displayed by means of graphical in the parameter display area. The time of current system dynamically display in the time display area. The function options of password access and parameter setting and the fault of query provided for users by touching graphical button in the function area. 
The Task of Active and Communication. The function of fault handler is called while system failure occurs mainly to implement the function that the communication with PC and drive the switch of system. The two tasks implemented by using the on-chip peripherals of GPIO and USART .The program call the corresponding library functions in accordance with the requirements in the design of configuration.

\section{The characteristics of the protector}

The chip can adopt sleep model, stop model or standby model to reduce the power consumption and prolong running time of equipment [6].In order to adjust the working condition according to the scene of the power supply system, the protector increased the protection function for signal-phase. The real-time multitasking operating system of UC/OS-III introduced in software, which realize the real-time protection. Through the testing capabilities provided by the real-time performance of the operating system during the development process of program combined with UC/Probe optimize the program code [7].

\section{Conclusion}

The protector adopts the chip of Cortex-M3 and administrates the software by the real-time operating system named UC/OS-III. With the gradually development of network of digital mine, the application of chip integrated the peripherals of Ethernet and WIFI can easy to achieve network function that expected to make intelligent protection function a further ascension.

\section{Acknowledgements}

This work was financially supported by Nation Natural Science Foundation( 61340015),Natural Science Research Project of Henan Province Education Department (2010A470003;2009B470003), International Science and Technology Cooperation Project of Henan Province(144300510014).

\section{References}

[1] Dongliang Yuan. Research on key technology of mine digital mine and its future development [J].Shandong Coal Science and Technology, (2014), (9):163-165(In Chinese).

[2] Jean J. Labrosse. UC/OS-III, The Real-Timer Kernel [M].Translator: Hui Gong, Ming Zeng and so on. Beijing: Beijing University of Aeronautics and Astronautics, (2014) (In Chinese).

[3] Huixiang Tang, Kun Hou and so on. Design of Intelligent Integrated Protective Unit for High-voltage Switch Based on RTOS [J].Colliery Mechanical\&ElectricalTechnology. (2008), (6):93-97(In Chinese).

[4] Hongtu Zhao, Shuping Chen, Yaohui WU. Analytical method of real sequence FFT algorithm using memory cell diagram [J], Computer engineering and design. (2012), 33(8): 3083-3088(In Chinese).

[5] Jianhong Li, Dan Liu, Zhan Xu. Research and Implementation of Extended Chinese Font Based on UCGUI Library [J].Computer Engineering.(2014),2(40): 271-274(In Chinese).

[6] Joseph Yin. The Definitive Guide to the ARM Cortex-M3 [M].Translator: Yan Song. Beijing: Beijing University of Aeronautics and Astronautics,(2011) (In Chinese).

[7] Jean J. Labrosse. UC/OS-III, The Real-Time Kernel for the STM32 ARM Cortex-M3 [M]. Translator: Xiaoqing He, Aihua Zhang. Beijing: Beijing University of Aeronautics and Astronautics, (2012) (In Chinese). 